\title{
Characterisation of superconducting capillaries for magnetic shielding of twisted-wire pairs in a neutron electric dipole moment experiment
}

\author{
S. Henry, M. Pipe, A. Cottle, C. Clarke, U. Divakar, A. Lynch \\ University of Oxford, Denys Wilkinson Building, Keble Road, Oxford OX1 3RH, UK.
}

\begin{abstract}
The cryoEDM neutron electric dipole moment experiment requires a SQUID magnetometry system with pick-up loops inside a magnetically shielded volume connected to SQUID sensors by long (up to $2 \mathrm{~m}$ ) twisted-wire pairs (TWPs). These wires run outside the main shield, and therefore must run through superconducting capillaries to screen unwanted magnetic pick-up. Superconducting capillaries can be produced by extruding the flux core of solder wire. After heating the solder wire in turpentine for an extended period of time, the flux is removed and a wire can be threaded through lengths of up to $1 \mathrm{~m}$. We show that the average measured transverse magnetic pick-up of a set of lengths of TWPs is equivalent to a loop area of $5.0 \times 10^{-6} \mathrm{~m}^{2} / \mathrm{m}$, or 14 twists per metre. From this we set the requirement that the magnetic shielding factor of the superconducting capillaries used in the cryoEDM system must be greater than $2.0 \times 10^{4}$. The shielding factor - the ratio of the signal picked-up by an unshielded TWP to that induced in a shielded TWP was measured for a selection of capillaries. We conclude the transverse shielding factor of a uniform capillary is greater than $10^{7}$. The measured pick-up was equal to, or less than that due to direct coupling to the SQUID sensor (measured without any TWP attached). We show that discontinuities in the capillaries substantially impair the magnetic shielding, yet if suitably repaired, this can be restored to the shielding factor of an unbroken capillary. We have constructed shielding assemblies for cryoEDM made from lengths of single core and triple core solder capillaries, joined by a shielded $\mathrm{Pb}$ cylinder, incorporating a heater to heat the wires above the superconducting transition as required.
\end{abstract}

\section{Introduction}

Measurements of the electric dipole moment (EDM) of the neutron are one of the most significant experimental tests of $\mathrm{T}$ (and hence $\mathrm{CP}$ ) violation, one of the factors required to explain the matter-antimatter asymmetry of the Universe. The current experimental limit of $\left|\mathrm{d}_{\mathrm{n}}\right|<2.9 \times 10^{-26} \mathrm{e} \cdot \mathrm{cm}$ set by the nEDM experiment [1] has already constrained, or ruled out, many theories. The next generation project, cryoEDM, aims to improve this limit by two orders of magnitude [2-5].

Magnetometry is an essential component of any neutron EDM experiment as the finite magnetic dipole moment of the neutron means magnetic field fluctuations between measurements of the precession frequency could mimic the frequency shift due to an electric dipole moment. To correct for this it is necessary to track changes in the magnetic field in the neutron cell between measurements to a precision of $\sim 0.1 \mathrm{pT}$. The nEDM experiment did this using a co-magnetometer which determined the magnetic field from the precession frequency of mercury nuclei in the neutron cell [6,7]. As this will not work at the $0.5 \mathrm{~K}$ temperature required for cryoEDM, we have developed a SQUID magnetometer for this purpose [8].

Figure 1 shows an overview of the cryoEDM apparatus. The neutron cells are located in the centre of a horizontal magnetic shield, made from multiple mu-metal layers and a superconducting $\mathrm{Pb}$ cylinder. The location of the SQUID sensors (outside the shield) and pick-up loops (close to the neutron cells) is shown. These must be separated by $\sim 2 \mathrm{~m}$ due to the high electric field in the neutron cell (which could induce a large voltage across the SQUID input, leading to permanent damage) and the distortion of the magnetic field by the SQUID sensors (due to the influence of the cryoperm shielding around the sensors, or the field produced by readout currents). The connection between the pick-up loops and the sensors is made by long NbTi twisted-wire pairs (TWPs). These wires must be magnetically shielded as they pass outside the main magnetic shield and would otherwise pick-up magnetic field fluctuations, which would mask the signal from the neutron cells $[9,10]$. Similar issues 
are faced by the SNS neutron EDM experiment magnetometer, although that is a different design, using SQUID gradiometers to measure the magnetic field from the precession frequency of ${ }^{3} \mathrm{He}$ nuclei [11].

This paper describes a superconducting magnetic shield developed for this purpose. This is made from lengths of $\mathrm{Pb}-\mathrm{Sn}$ solder, where the flux core has been removed creating a hollow capillary. The following sections describe the requirements for the experiment and the design of our system. Section 5 describes the production of the solder capillaries and section 6 gives the details and results of experimental tests conducted to measure the pick-up of TWPs and the shielding factor of the capillaries. Section 7 shows frequency spectra recorded by shielded and unshielded TWPs and confirms that the capillaries can be used to reduce the noise from external magnetic field fluctuations to the intrinsic SQUID noise. Section 8 gives the results of tests of the heaters incorporated into the shielding assembly.

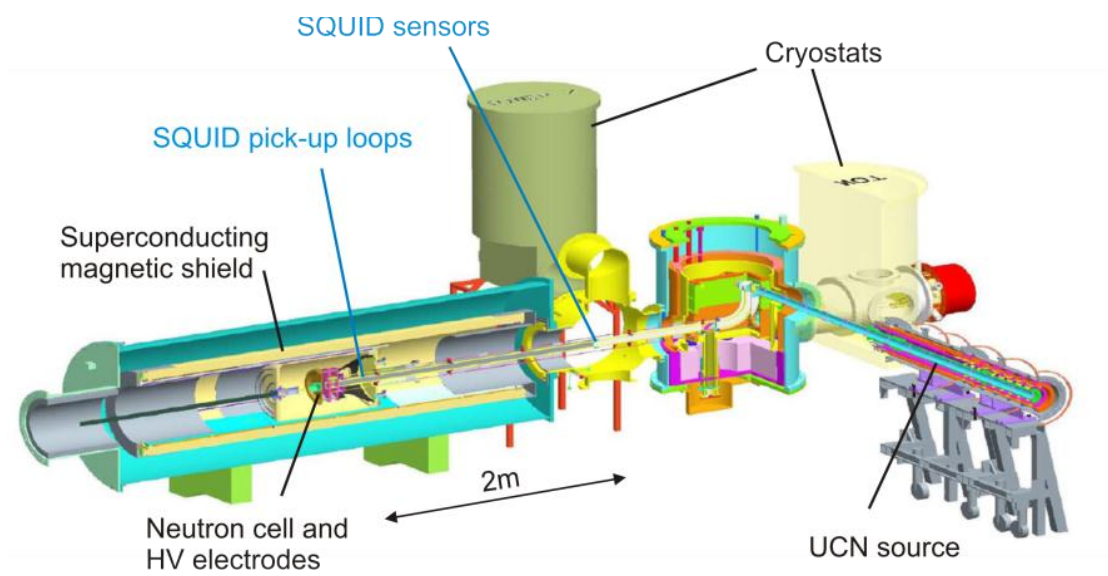

Figure 1 An overview of the cryoEDM apparatus showing the main components and the location of the SQUID sensors and pick-up loops. The neutron beam from the ILL reactor enters from the right into the superthermal ultra-cold neutron (UCN) source. The neutrons are stored for the Larmor frequency measurement in neutron cells at the centre of the superconducting magnetic shield.
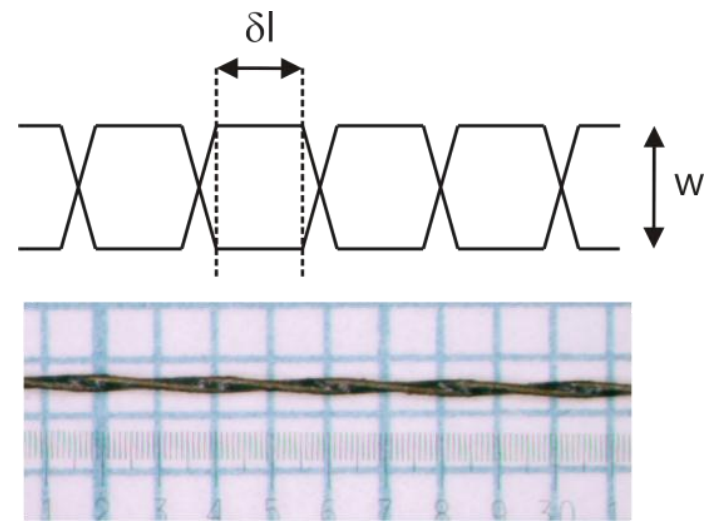

Figure 2. A simple model of twisted-wire-pair geometry and a photograph of a TWP used for these tests (coated in Stycast after twisting). The average twist length was $\delta \mathrm{l}=\mathbf{2 . 7} \mathbf{m m}$, and the wire diameter $w=0.127 \mathrm{~mm}$. 


\section{Twisted-wire pairs}

Twisting two wires together is a well-established technique to minimise susceptibility to unwanted magnetic signals. The principle is that the magnetic flux through two adjacent loops created by the twisting will cancel, so there is no net magnetic pick-up. In this section we first calculate the signal induced in an ideal TWP in a uniform field, and a field with a constant gradient. As the measured pick-up is much larger than these values, we conclude that a real TWP deviates from a perfect twisting, and these imperfections are responsible for most of the pick-up.

\subsection{Theoretical magnetic pick-up in uniform field}

The current induced in a superconducting loop due to a change in the magnetic flux $\Phi$ is given by

$$
\Delta i=\frac{\Phi}{L},
$$

where $\mathrm{L}$ is the inductance of the circuit. In a SQUID magnetometer circuit

$$
\Phi=\delta B_{s} A+\delta B_{e} \delta A
$$

where $\delta \mathrm{B}_{\mathrm{S}}$ is the magnetic field fluctuation at the neutron cell, which we wish to measure using a pick-up loop of area $\mathrm{A}$, and $\delta \mathrm{A}$ is the additional loop area formed by the readout wires between the SQUID input and the pick-up loop, which is exposed to magnetic fluctuations $\delta \mathrm{B}_{\mathrm{e}}$. In a well-designed system, we aim to make $\delta \mathrm{A}$ as small as possible.

In a simple model, a TWP is treated as a series of rectangular loops in a flat plane, oriented in alternating directions as shown in Figure 2 [12]. Although a real TWP is not flat, this is a fair approximation of the area as seen from one direction. A perfect TWP exposed to a spatially uniform magnetic fluctuation $\delta \mathrm{B}_{\mathrm{e}}$ will only give a signal if there are an odd number of loops. The signal in this case, on a calibrated magnetometer, is then

$$
\Delta S_{e}=\delta B_{e} \frac{\delta A}{A},
$$

where $\delta A$ is the area of a single twist. For wire of diameter $\mathrm{w}=0.127 \mathrm{~mm}$, and a twist length $\delta \mathrm{l}=2.7 \mathrm{~mm}$, we estimate a maximum loop area of $\delta A=w \delta l$. Thus for a $20 \mathrm{~mm}$ diameter pick-up loop (the smallest loop size used in the cryoEDM system), a magnetic fluctuation of $\delta B=1 \mu T$ along the TWP would give an error signal of $273 p T$.

\subsection{Theoretical pick-up in field with finite gradient}

If the magnetic field fluctuations are not spatially uniform, but have a finite gradient, this will give a net pick-up as the flux through each twist is slightly different from that through adjacent loops. This will be significant in the cryoEDM experiment where the TWPs run into the main magnetic shield, so the magnitude of field fluctuations will fall from their full external magnitude to close to zero along the length. For a magnetic field with a fixed gradient given by

$$
B(x)=B_{0}+\frac{d B}{d x} x
$$

applied along a TWP with an even number of twists N, over length L, will give a net flux of 


$$
\begin{aligned}
& \Phi=\sum_{i=1}^{N / 2} B\left(\frac{2 i+1}{N} L\right) \delta A-B\left(\frac{2 i}{N} L\right) \delta A \\
& =\sum_{i=1}^{N / 2}\left(\frac{d B}{d x}\right) \frac{L}{N} \delta A \\
& =\delta A\left(\frac{d B}{d x}\right) \frac{L}{2}
\end{aligned}
$$

We define a twist as a $180^{\circ}$ rotation, exchanging the positions of the two wires. For a field gradient of $2 \mu \mathrm{T} / \mathrm{m}$ along $0.5 \mathrm{~m}$, we estimate a maximum error signal due to this effect of magnitude 136pT.

\subsection{Magnetic pick-up of real twisted-wire pair}

In this paper we will show that the measured pick-up in a twisted-wire pair exposed to a magnetic signal is greater than these theoretical values. This is because the most significant cause of magnetic pick-up is due to imperfections in the twisting, as the precise size and shape of each twist will vary along the length. This produces a net flux through the TWP that is much larger than the effects described above. This effect cannot be calculated without precise information on the geometry of the wires, therefore we measure the pick-up directly, as outlined in Section 6, and characterise it in terms of an effective area $\delta \mathrm{A}$ of the TWP per metre. We measure an effective area of $5 \times 10^{-6} \mathrm{~m}^{2} / \mathrm{m}$, giving an error signal of $2.0 \mathrm{nT}$ in the cryoEDM setup due to a $1 \mu \mathrm{T}$ fluctuation, without the capillary shielding.

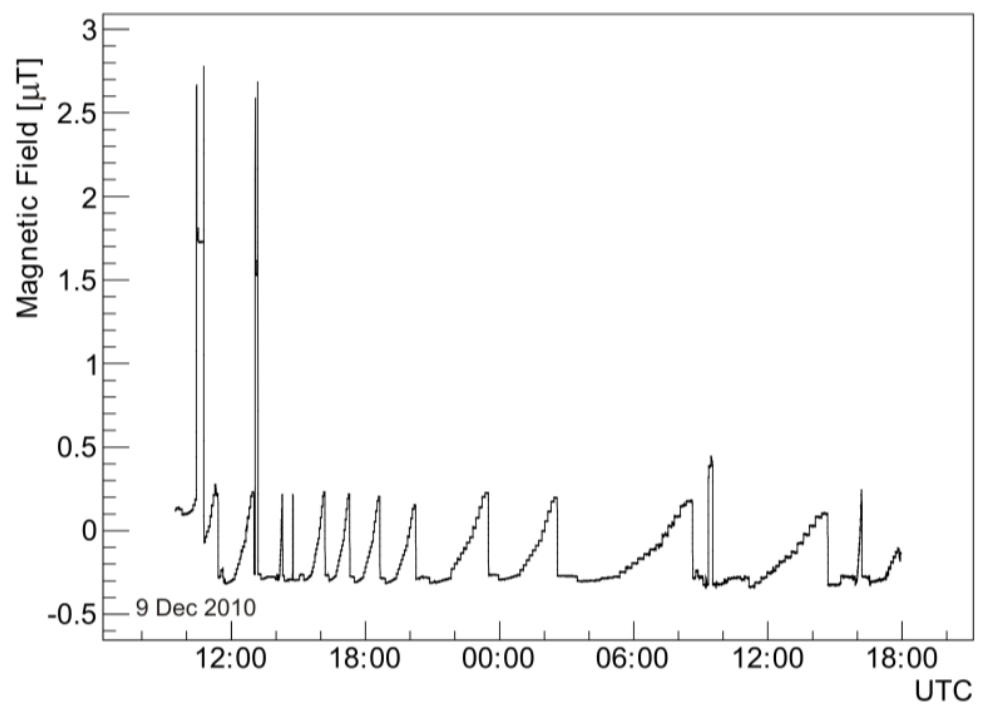

Figure 3 The magnetic field variation recorded over a 32 hour period by a fluxgate magnetometer by the cryoEDM experiment in hall ILL22 at the Institut-Laue Langevin. The saw tooth features are due to changes in the magnetic field generated at the IN15 neutron spin echo spectrometer [13]. The larger glitches are due to movement of the overhead crane.

\section{Magnetic shielding requirements for SQUID connections}

A neutron EDM measurement is carried out using the Ramsey resonance technique, in which a large number of ultra-cold neutrons are stored inside a magnetic and electric field, and their Larmor spin precession frequency is measured. A non-zero neutron EDM will produce a shift in the precession frequency when the electric field is reversed. As the neutron has a non-zero magnetic dipole moment, a change in the magnetic field will produce the same effect. 
It can be shown that a change in the magnetic field of just 4.3fT is equivalent to a false EDM signal at the level of the current limit [1], in an electric field of the magnitude used in that experiment. This represents the difference between the average magnetic field over all runs with parallel $\mathrm{E}$ and $\mathrm{B}$ fields, and that with antiparallel fields. To achieve this it is necessary to control and monitor the magnetic field between each run to a precision of $\sim 0.1 \mathrm{pT}$.

Figure 3 shows the variation in the magnetic field recorded by a fluxgate magnetometer in the ILL experiment hall over a 32-hour period. Typical short-term magnetic fluctuations in the experimental hall at ILL are below $1 \mu \mathrm{T}$. Occasional larger disturbances can be identified from the magnetometer data, and these periods excluded from the neutron EDM analysis. Magnetic shielding for the neutron cells is achieved using a combination of mu-metal and superconducting $\mathrm{Pb}$ cylinders. The SQUID pick-up loops are located at the centre of this shielding, and are connected to the SQUID sensors, which are mounted in an unshielded part of the cryostat $\sim 0.5 \mathrm{~m}$ away from the end of the main shield.

The magnetic shielding factor for the TWP cannot be measured in the same way as a conventional magnetic shield as the core is so narrow we cannot measure the field at a defined point in the centre. Instead we define the shielding factor as the ratio of the signal induced in the TWP without the shield, to that measured with the shield. To determine the required shielding factor we use the effective area per metre of our TWP, measured as $\delta A=5 \times 10^{-6} \mathrm{~m}^{2} / \mathrm{m}$ (see section 6). The noise signal on the $20 \mathrm{~mm}$ diameter pick-up loops in the cryoEDM system, created by a magnetic field change of $\Delta B=1 \mu \mathrm{T}$ due to pick-up along the $\mathrm{l}=0.5 \mathrm{~m}$ section of TWP outside the main magnetic shield is then given by

$$
\Delta S=\delta B \frac{\delta A \cdot l}{A} \frac{1}{F}=\frac{2000 p T}{F}
$$

where $\mathrm{F}$ is the shielding factor provided by the superconducting capillary. Therefore to achieve our target resolution of $0.1 \mathrm{pT}$, we need a shielding factor of at least 20,000.

This full shielding factor is required for the first $0.3 \mathrm{~m}$, which is outside the main magnetic shield. Once the TWPs move into the main magnetic shield, the magnitude of the field fluctuations will reduce, but shielding is still required for a length of total $1.2 \mathrm{~m}$ to ensure the system measures the field at the location of the pick-up loop and not along the TWP.

\section{Superconducting capillary shield design for TWPs in the cryoEDM experiment}

The requirement for the cryoEDM experiment was magnetic shields, of length $1.2 \mathrm{~m}$, for twelve twisted pairs of $0.127 \mathrm{~mm}$ diameter NbTi wire. Superconducting niobium capillaries can also be used for screening twisted-wire-pairs [14]. However we decided to use the solder alloy, which could be bent into the necessary shapes without damage to either the capillary or TWP. Trial experiments using the method described in section 5 showed that we could remove the flux from $1 \mathrm{~mm}$ diameter single core solder wire for lengths up to $0.3 \mathrm{~m}$. Lengths of up to $1 \mathrm{~m}$ could be produced from thicker $2 \mathrm{~mm}$ solder wire with a triple core. The final shielding design uses a combination of single and triple core lengths.

The shielding assembly is illustrated in Figure 4. Four such assemblies each carry three TWPs to provide connections for the twelve SQUID channels. After the flux was removed from a $\sim 0.9 \mathrm{~m}$ length of $2 \mathrm{~mm}$ diameter three-core solder wire, a TWP was threaded through each of the cores. Each of these pairs was then threaded through a $0.3 \mathrm{~m}$ length of $1 \mathrm{~mm}$ solder wire. The join between the sections was shielded using a $5.2 \mathrm{~mm}$ outer diameter $\mathrm{Pb}$ cylinder, made from two parts soldered together. This contained a $390 \Omega$ resistor connected to a separate wire pair, which was used as a heater to heat the superconducting wires into their conducting state when necessary. 
The heater cylinder was filled with Stycast epoxy to insulate the resistor and wires from the superfluid helium. The $\mathrm{Pb}$ cylinder was wrapped in a layer of $\mathrm{Pb}$ foil, and surrounded by an outer cylinder moulded from Stycast to provide addition thermal insulation and to protect the assembly.

An advantage of using solder, instead of another material such as $\mathrm{Nb}$, for such superconducting capillaries is that the solder is too soft to damage the HML insulation layer on the wires. All the shielded TWP assemblies constructed were tested for electrical shorts between the NbTi wire and the solder shield, but none were found.
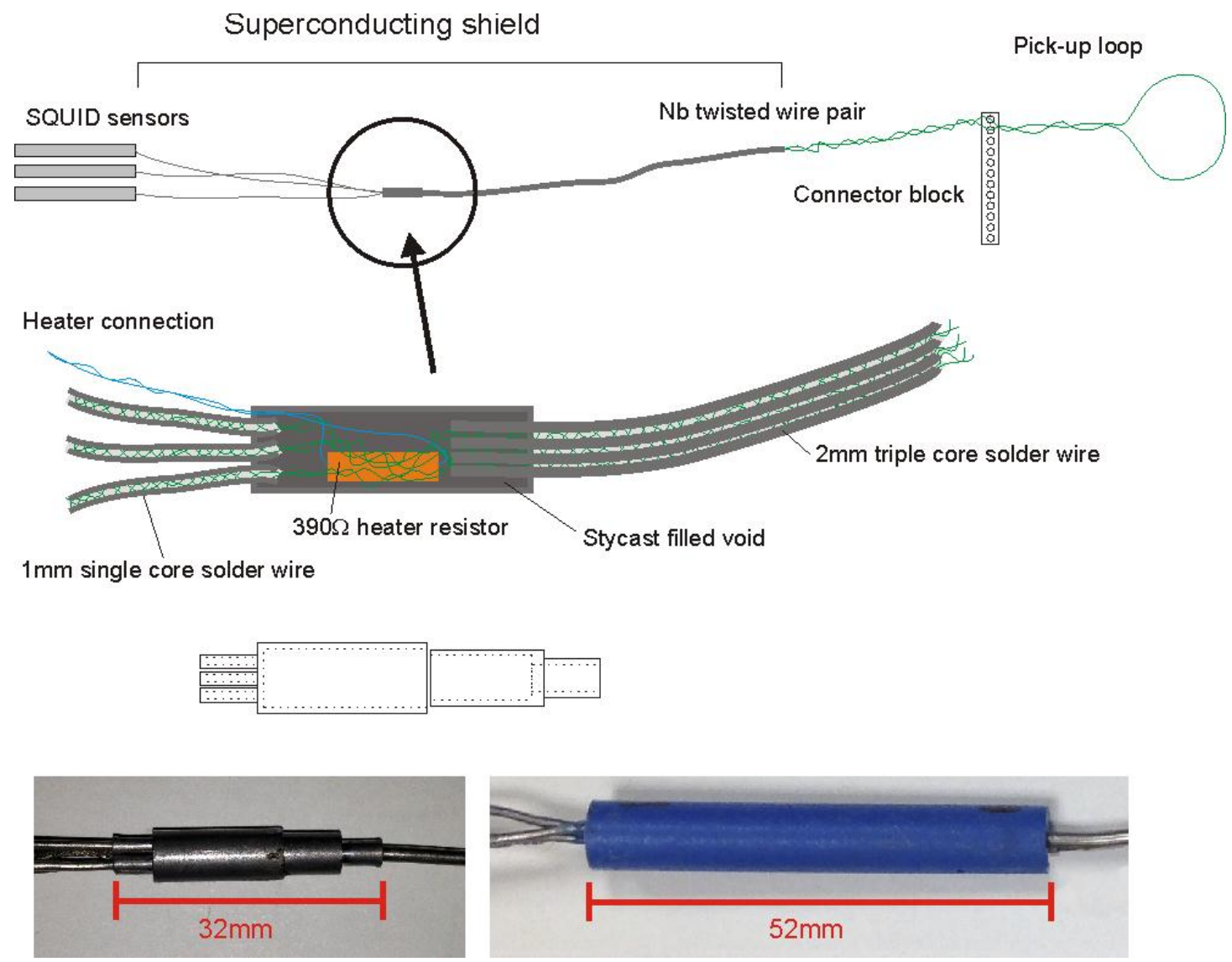

Figure 4. Illustration of the superconducting shield for the twisted wire pairs, including the heater resistor. The single and triple core solder lengths are joined by two machined lead pieces soldered together. A photograph of a prototype piece is shown. After assembly as shown, the heater piece is wrapped in an extra layer of $\mathrm{Pb}$ foil and surrounded by an outer jacket of Stycast to give the final heater assembly as shown in the right photograph.

\section{Production of solder capillaries}

To remove the flux core from electrical solder wire, the wire lengths were left in hot turpentine for an extended period. Turpentine has a boiling point of $149-180^{\circ} \mathrm{C}$ [15], slightly below the melting point of solder $\left(183-188^{\circ} \mathrm{C}[16]\right)$. By placing lengths of solder wire in turpentine, and heating to just below the boiling point $\left(165-170^{\circ} \mathrm{C}\right)$ for a period of $>24$ hours, the flux will dissolve in the turpentine. Figure 5 shows a diagram of the apparatus used. The evaporated turpentine is recovered using a distillation apparatus. After being used several times, the turpentine can become saturated with flux; in this case it can then be purified by running the setup at a higher temperature, without any solder, to distil the oil.

An alternative method to remove the flux is described in [17]. 


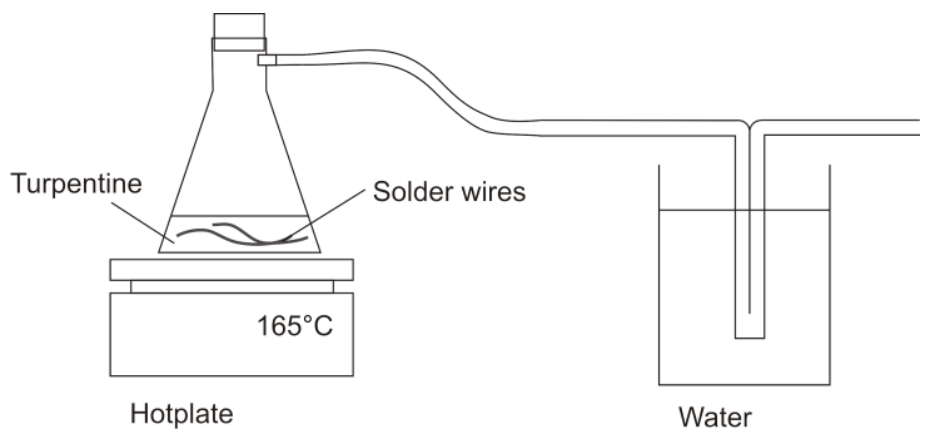

Figure 5. Diagram of the apparatus used to heat the solder wires in turpentine and collect the evaporated oil.

\section{Measurement of the magnetic pick-up of TWPs and magnetic shielding factor of the capillaries}

In order to test the design to ensure it met the required shielding factor for cryoEDM, and to investigate the magnetic shielding provided by such capillaries we carried out a series of tests. Figure 6 shows a drawing of the six-SQUID assembly built to measure the TWP magnetic pick-up and the shielding factor of the solder capillaries. This used a 3-axis Helmholtz coil to apply a magnetic signal of any direction to the twisted wire pairs connected to SQUID inputs. The TWPs were terminated inside the capillary with a tiny loop $\sim 20 \mathrm{~mm}$ from the end of the shield. They were coated with a thin layer of Stycast to prevent the wires from unravelling between measurements. This assembly was mounted on a dipstick, which could be lowered into a cryostat with an external mu-metal shield to reduce the external magnetic fluctuations. The SQUID system was the same as that used for cryoEDM: sensors supplied by Supracon, and the readout used a PFL800 preamplifier from STAR Cryoelectronics with control and digitisation electronics developed in house.

The SQUID sensors themselves were shielded from the magnetic field by a $22 \mathrm{~mm}$ length niobium cylinder, inside a $80 \mathrm{~mm}$ Cryoperm shield. When installed, the solder capillaries ran into the Cryoperm, right up to the niobium can.
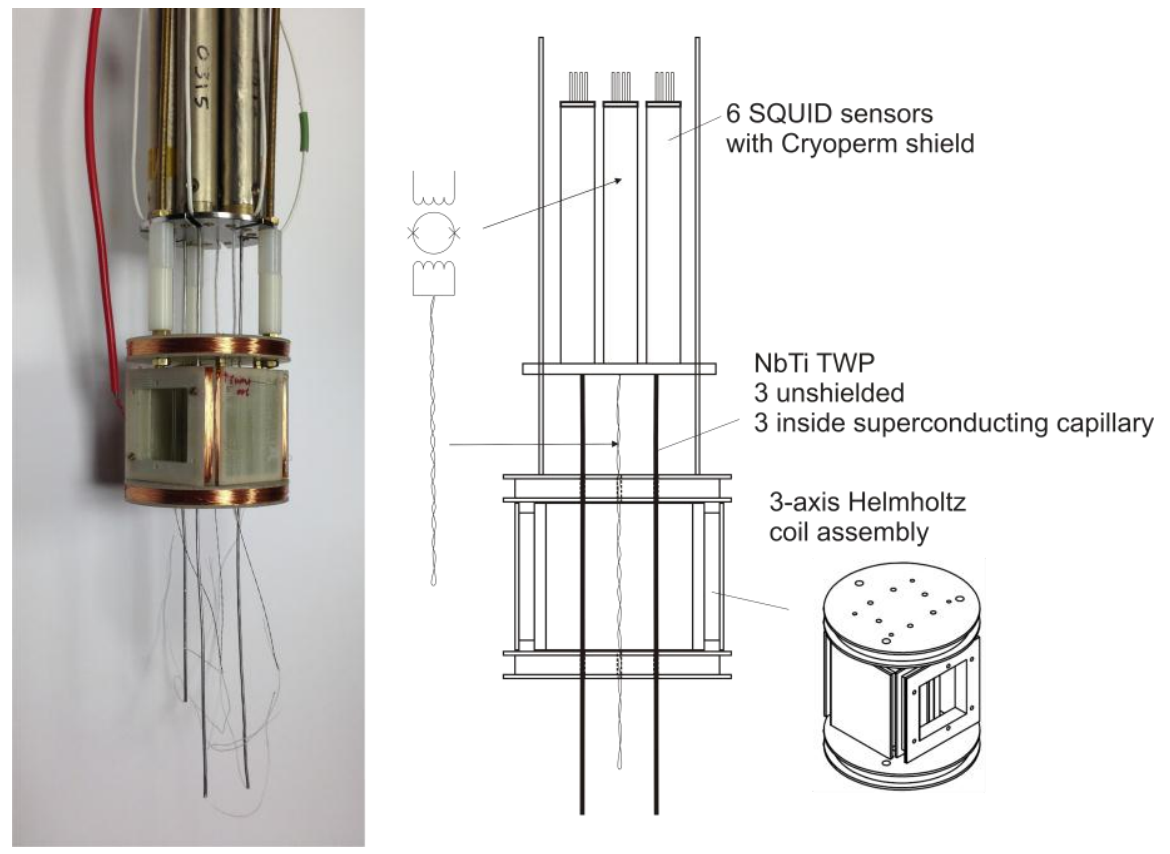

Figure 6. The test setup used to measure the shielding factor of the superconducting capillaries. The shielded twisted wire pairs connected to the SQUID inputs were positioned inside a Helmholtz coil set to generate a magnetic signal of defined direction. This assembly was mounted on a dipstick and lowered into a cryostat of liquid helium. 
The TWP pick-up was measured by applying a magnetic field step of known magnitude to an unshielded TWP. The capillary shielding factor was measured by recording the signals on the SQUIDs connected to shielded and unshielded TWPs when a current was applied to the Helmholtz coils. The capillaries were then moved to the previous unshielded channels and the measurements repeated. We then compared the signals measured on the same TWP with and without the capillary shield (normalised to the applied current).

\subsection{TWP magnetic pick-up measurements}

To determine the effective area of the TWP, used in Equation 1, we need to measure the signal induced on the SQUID due to a magnetic field step applied to the twisted wires. The magnitude of this field is calculated by numerically integrating the magnetic field from the coils along the length of the TWP. We define the twisted wire pair pick-up factor

$$
P=\frac{\Delta S_{\text {test }}}{\Delta B_{\text {test }} l_{\text {test }}}=\frac{\Delta S_{\text {test }}}{\int_{0}^{L} \Delta B_{\text {test }}(I) d l}
$$

From this we can calculate the effective area as

$$
\delta A=P L C
$$

Where $\mathrm{L}$ is the inductance of the input circuit (the SQUID input inductance of $350 \mathrm{nH}$, plus that of the TWP sample, calculated as $324 \mathrm{nH} / \mathrm{m}$, using the equation for a pair of long parallel wires $[18,19]) ; \mathrm{C}$ is a calibration factor to convert the SQUID output signal to the current through the input coil.

$$
C=\frac{0.2 \mu A / \Phi_{0}}{3.7 V / \Phi_{0}}
$$

We measured the pick-up and shielding factors for the axial $(\mathrm{Z})$ and transverse directions. Generally speaking, the symmetry of a TWP (in general) and shield mean it is not beneficial to distinguish the two transverse axes (here labelled $\mathrm{X}$ and $\mathrm{Y}$ ) when quoting results. However in each measurement the $\mathrm{X}$ and $\mathrm{Y}$ pick-up could be different due to asymmetries in the twisting of an individual TWP. Therefore we designed the apparatus with three Helmholtz coils to allow a magnetic field to be applied in turn to the $\mathrm{X}, \mathrm{Y}$ and $\mathrm{Z}$ directions during each cooldown. We then define the transverse pick-up as $\sqrt{P_{X}^{2}+P_{Y}^{2}}$

The magnetic field signal along the length of the TWP, due to a current applied to the Helmholtz coils was calculated by numerically integrating the Biot-Savart law. Away from the centre of coils, this includes a small off-axis field. This was accounted for by applying a matrix transformation to the pick-up signals measured due to a current applied to each coil. So equation 2 becomes:

$$
\left(\begin{array}{l}
P_{x} \\
P_{y} \\
P_{z}
\end{array}\right)=\left(\begin{array}{lll}
B l_{x}^{1} & B l_{y}^{1} & B I_{z}^{1} \\
B I_{x}^{2} & B l_{y}^{2} & B l_{z}^{2} \\
B l_{x}^{3} & B l_{y}^{3} & B I_{z}^{3}
\end{array}\right)^{-1}\left(\begin{array}{l}
S_{1} \\
S_{2} \\
S_{3}
\end{array}\right)
$$

where $S_{1,2,3}$ is the signal recorded on a SQUID due to a current applied to Helmholtz coils 1,2,3 (aligned with the $\mathrm{x}, \mathrm{y}, \mathrm{z}$ axes). The matrix elements give the integrated field (nT·m) along the length of the TWP for each field component,

$$
B I_{x, y, z}^{1,2,3}=\int_{0}^{L} \Delta B(I)_{x, y, z}^{1,2,3} d l
$$

calculated for each coil. The off-diagonal elements are small. 
Using our model of the field generated by the Helmholtz coils, we carried out a numerical simulation of our test setup, calculating the flux through each twist due to the field at that point and summing this up. This gave a theoretical pick-up factor (assuming uniform loop sizes) of $0.012 \mathrm{~V} / \mathrm{nT} \cdot \mathrm{m}$. The represents the pick-up expected due to the coupling discussed in Sections 2.1 and 2.2 .

The pick-up was measured using a selection of $0.23 \mathrm{~m}$ twisted wire pairs made from $0.127 \mathrm{~mm}$ diameter NbTi. The results are given in Table 1. As each TWP has slightly different variations in twist size, we expect this to vary from sample to sample. The average pick-up measured for transverse fields was $0.20 \mathrm{~V} / \mathrm{nT} \cdot \mathrm{m}$, with a standard deviation over 6 samples of $0.1 \mathrm{~V} / \mathrm{nT} \cdot \mathrm{m}$. This corresponds to an effective area of $5 \times 10^{-6} \mathrm{~m}^{2} / \mathrm{m}$ (equivalent to the area of 14 twists per metre). The axial pick-up (which in the model is zero) is a factor of 20 lower.

\begin{tabular}{|l|l|l|}
\hline \multirow{2}{*}{ Sample } & \multicolumn{2}{|l|}{ Pick-up factor $[\mathrm{V} / \mathrm{nT} \cdot \mathrm{m}]$} \\
\cline { 2 - 3 } & Transverse & Axial \\
\hline TWP 1 & $0.30 \pm 0.06$ & $0.011 \pm 0.002$ \\
\hline TWP 2 & $0.25 \pm 0.07$ & $0.015 \pm 0.005$ \\
\hline TWP 3 & $0.08 \pm 0.01$ & $0.003 \pm 0.002$ \\
\hline TWP 4 & $0.30 \pm 0.01$ & $0.015+0.017$ \\
\hline TWP 5 & $0.10 \pm 0.02$ & $0.006 \pm 0.006$ \\
\hline TWP 6 & $0.14 \pm 0.05$ & $0.008 \pm 0.006$ \\
\hline
\end{tabular}

Table 1 The pick-up factor measured for a selection of twisted-wire-pairs for magnetic signals applied in the transverse and axial directions. The errors show the standard deviation of measured values over multiple measurements during separate cooldowns.

\subsection{Magnetic shielding factor of superconducting capillaries}

The magnitude of a transverse magnetic signal applied to a uniform superconducting tube of radius $a$ is attenuated by a factor of $\exp \left(-1.84 \frac{z}{a}\right)$ at a distance $\mathrm{z}$ inside the tube [20]. From this we expect the magnetic field inside a superconducting capillary to be negligible once we move more than a few $\mathrm{mm}$ from the end. The pick-up of a TWP may be increased due to the gradient of the field inside the capillary, and if the solder is not a uniform superconductor. However it seems reasonable to expect that unless the capillary is damaged, the shielding factor will be very large and what we measure will be limited by the magnetic pick-up at the point where the TWP leaves the capillary and is connected to the SQUID input.

The measured values of the shielding factors (SF) are given in Table 2. These were calculated by dividing the pick-up factor measured for an unshielded TWP by that measured for the same TWP with a solder shield in place. The shield was then removed and the test repeated. These tests were repeated with a selection of different shields made from single core and triple core solder. The transverse shielding factor measured for unbroken capillaries was always $>10^{6}$.

To establish the limit of our measurement apparatus, we carried out a control test, applying an external field to a setup with the TWP removed and the SQUID input shorted with a superconducting link. With this, we measured a pick-up of a similar magnitude to the measurements with a shielded TWP. This represents the magnetic coupling to the SQUID itself. The spread of values for repeated measurements with each capillary sample shows that the measured pick-up is highly sensitive to the small changes introduced when the TWP was fitted or removed from a capillary and SQUID. The highest value measured for the transverse shielding factor for all samples was greater than $10^{7}$. We take this as a lower limit on the shielding factor of a solder capillary. 


\begin{tabular}{|l|l|l|l|}
\hline Label & Description & \multicolumn{2}{|l|}{$\begin{array}{l}\text { Minimum and maximum magnetic } \\
\text { shielding factor measured in multiple } \\
\text { tests }\end{array}$} \\
\cline { 3 - 4 } & & Transverse & Axial \\
\hline SCC \#1 & $\begin{array}{l}\text { Single core solder, 1mm } \\
\text { diameter, 240mm length }\end{array}$ & $(1.5-4.0) \times 10^{7}$ & $(0.5-3.3) \times 10^{6}$ \\
\hline SCC \#4 & $\begin{array}{l}\text { Single core solder, 1mm } \\
\text { diameter, 260mm length }\end{array}$ & $(0.6-24) \times 10^{7}$ & $(1.1-54) \times 10^{6}$ \\
\hline SCC \#5 & $\begin{array}{l}\text { Single core solder, 1mm } \\
\text { diameter, 260mm length }\end{array}$ & $(0.8-4.2) \times 10^{7}$ & $(1.0-66) \times 10^{6}$ \\
\hline SCC \#9 & $\begin{array}{l}\text { Triple core solder, 2mm } \\
\text { diameter, }\end{array}$ & $(6.0-24) \times 10^{7}$ & $(3.3-12) \times 10^{7}$ \\
\hline SCC \#10 & $\begin{array}{l}\text { Triple core solder, 2mm } \\
\text { diameter, }\end{array}$ & $(1.7-1.8) \times 10^{7}$ & $(0.1-2.9) \times 10^{6}$ \\
\hline Control & $\begin{array}{l}\text { No TWP attached, } \\
\text { SQUID input shorted }\end{array}$ & $(0.8-2.2) \times 10^{7}$ & $(0.5-5.7) \times 10^{6}$ \\
\hline
\end{tabular}

Table 2 Shielding factors measured for the superconducting capillary (SCC) samples, by comparing the pick-up with and without the capillary. These values should be taken as lower limits, as the magnitude is comparable to the control test with no TWP attached. The control test was repeated with three different SQUIDs, comparing the pick-up of an unshielded TWP with a SQUID with shorted input.

\begin{tabular}{|c|c|c|c|}
\hline \multirow[t]{2}{*}{ Label } & \multirow[t]{2}{*}{ Description } & \multicolumn{2}{|c|}{$\begin{array}{l}\text { Minimum and maximum magnetic } \\
\text { shielding factor measured in multiple } \\
\text { tests }\end{array}$} \\
\hline & & Transverse & Axial \\
\hline SCC \#6 & $\begin{array}{l}\text { Single core solder, } 1 \mathrm{~mm} \\
\text { diameter, } 255 \mathrm{~mm} \text { length, } \\
\text { broken in centre }\end{array}$ & $(1.1-1.7) \times 10^{1}$ & $(7.6-19) \times 10^{0}$ \\
\hline SCC \#7 & $\begin{array}{l}\text { Single core solder, } 1 \mathrm{~mm} \\
\text { diameter, } 255 \mathrm{~mm} \text { length, } \\
\text { broken in centre }\end{array}$ & $(4.4-6.7) \times 10^{0}$ & $(3.6-180) \times 10^{0}$ \\
\hline$\overline{\text { SCC \#8 }}$ & $\begin{array}{l}\text { Single core solder, } 1 \mathrm{~mm} \\
\text { diameter, } 255 \mathrm{~mm} \text { length, } \\
\text { broken in centre }\end{array}$ & $(6.2-13) \times 10^{0}$ & $(9.2-24) \times 10^{0}$ \\
\hline SCC \#6.1 & $\begin{array}{l}\text { \#6 repaired with one layer } \\
\mathrm{Pb} \text { foil }\end{array}$ & $3.7 \times 10^{2}$ & $2.3 \times 10^{1}$ \\
\hline SCC \#7.1 & $\begin{array}{l}\text { \#7 repaired with one layer } \\
\mathrm{Pb} \text { foil }\end{array}$ & $(0.9-4.4) \times 10^{2}$ & $(3.5-50) \times 10^{0}$ \\
\hline SCC \#8.1 & $\begin{array}{l}\text { \#8 repaired with one layer } \\
\mathrm{Pb} \text { foil }\end{array}$ & $(4.0-8.6) \times 10^{2}$ & $(1.3-3.4) \times 10^{1}$ \\
\hline SCC \#6.2 & $\begin{array}{l}\# 6 \text { repaired with several } \\
\text { layers } \mathrm{Pb} \text { foil }\end{array}$ & $(0.3-57) \times 10^{4}$ & $(0.5-230) \times 10^{3}$ \\
\hline SCC \#7.2 & $\begin{array}{l}\# 7 \text { repaired with several } \\
\text { layers } \mathrm{Pb} \text { foil }\end{array}$ & $(0.7-14) \times 10^{3}$ & $(0.2-4.0) \times 10^{3}$ \\
\hline SCC \#8.2 & $\begin{array}{l}\text { \#8 repaired with several } \\
\text { layers } \mathrm{Pb} \text { foil }\end{array}$ & $(0.8-23) \times 10^{4}$ & $(0.8-25) \times 10^{4}$ \\
\hline SCC \#11 & $\begin{array}{l}\text { Single core solder, } 1 \mathrm{~mm} \\
\text { diameter, } 255 \mathrm{~mm} \text { length, } \\
\text { broken in centre repaired } \\
\text { by soldering }\end{array}$ & $(2.5-3.8) \times 10^{6}$ & $(1.1-2.4) \times 10^{6}$ \\
\hline
\end{tabular}

Table 3 Shielding factor measurements for capillaries which had been deliberately broken, and then repaired. For each repeated measurement, the capillary was removed from the TWP, and then reinstalled, and re-repaired. 
The axial shielding factors showed a greater spread of values, and were sometimes lower than the transverse factors, although as the transverse pick-up factors are much greater than the axial, the transverse SF is our primary concern.

After measuring the shielding factors of the continuous capillaries, we repeated the test using capillaries formed of two sections. Such breaks may occur if a solder capillary is subjected to stress at cryogenic temperatures. We first measured the SF with the two pieces simply pushed together, and then 'repaired' the join using one or more layers of $\mathrm{Pb}$ foil; and in one case, using a soldering iron. The results of these tests are given in Table 3

This showed that discontinuities in the superconducting capillaries reduced the shielding factors by several orders of magnitude to levels which would be unsatisfactory for the cryoEDM experiment. However, when repaired with several layers of $\mathrm{Pb}$ foil, the shielding factor increased, with varying levels of success. Soldering capillaries together resulted in shielding factors consistent with continuous shields. However attempting such a repair carries a risk of creating further damage.

We conclude that the magnetic shielding provided by the solder capillaries meets the requirements of our system provided the capillaries are continuous. If a capillary is broken, repair is possible, but should be done with care and tested to ensure the shielding factor is not compromised, and it would be better to replace the shield where possible.

\section{Noise spectra}

As a further test of the shielding properties of the superconducting capillaries, we recorded frequency spectra of a set of SQUIDs connected to shielded and unshielded TWPs, and with the TWPs replaced by a short across the sensor input. These were measured in an unshielded dewar in our Oxford laboratory. The spectra are shown in Figure 7. This shows that while the unshielded TWP picks up a high level of noise, the level of magnetic field fluctuations measured with a shielded TWP is attenuated to the level of the intrinsic SQUID noise.

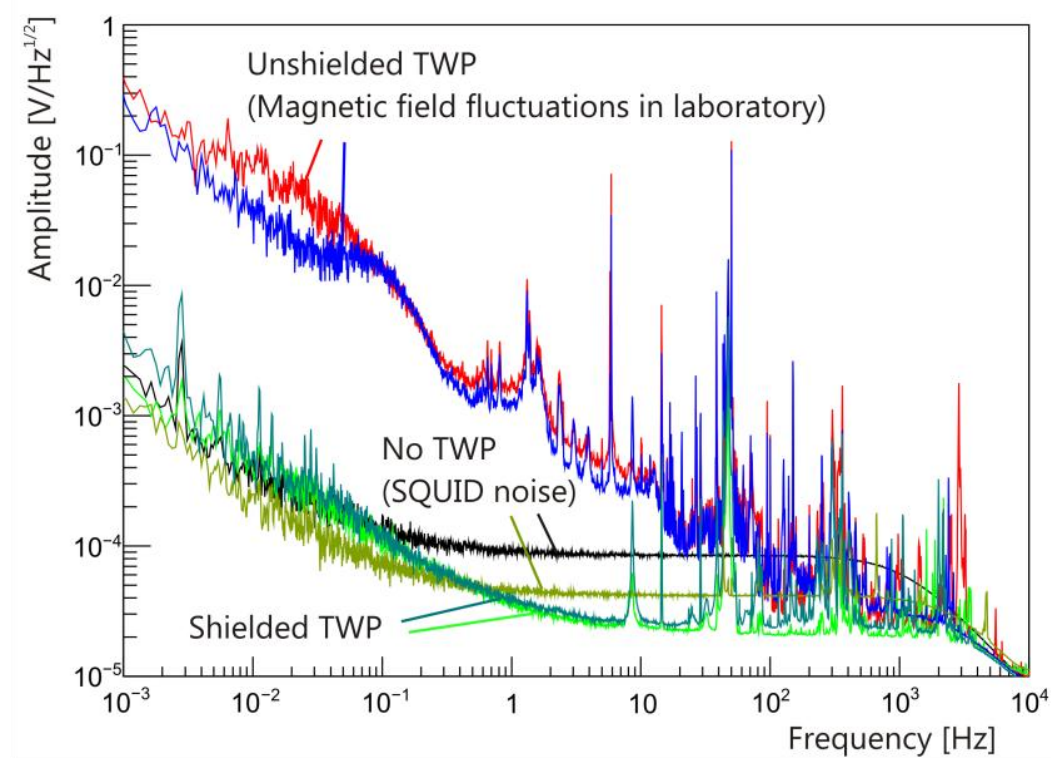

Figure 7 Frequency spectra recorded with SQUIDs connected to: TWPs shielded with superconducting capillaries (green, teal); unshielded TWPs (red, blue); and with shorted inputs (black, olive). The SQUIDs connected to unshielded TWPs measure the magnetic noise in the laboratory. The bare SQUIDs measure the intrinsic SQUID noise. The capillaries reduce the measured noise to the level of the SQUID noise below $1 \mathrm{~Hz}$. The white noise level is larger for the shorted input SQUIDs, as this level varies with the impedance connected across the sensor input [21]. 
The white noise level of the shorted input SQUID, which dominates above $1 \mathrm{~Hz}$, is actually greater than that recorded with the shielded TWP attached, as the inductance on the input is different, which affects the level of the white noise [21]. This also varies from sensor to sensor. These tests were carried out at in liquid helium at $4.2 \mathrm{~K}$. In the cryoEDM system the SQUIDs will run in superfluid helium at $0.5 \mathrm{~K}$, therefore we expect the white noise level will be reduced by a factor of $\sqrt{\frac{4.2}{0.5}}=2.9$.

The spectra for shielded TWPs show some noise peaks, most notably for $50 \mathrm{~Hz}$ and harmonics, which were not present for the bare SQUIDs. This is not so important for a neutron EDM experiment, as we are interested in the signal averaged over a long time period and this can be removed with digital filters. However we note that the superconducting shield may not provide such good shielding for higher frequency signals.

\section{Heater Tests}

The heater assembly described in Section 4 is designed to allow the heating of the three NbTi TWPs above their superconducting transition upon application of a control current. In the cryoEDM apparatus, the heater, with the connections and filters, has a total resistance of $1 \mathrm{k} \Omega$, and the DAC driving the heaters had a maximum output of $20 \mathrm{~V}$, giving a maximum possible power of $156 \mathrm{~mW}$. Therefore the heaters must be able to heat the wire into a conducting state with a power of this, or less. The power required to break the superconductivity for two heaters is given in Table 4; all the heaters meet this requirement, with an average power requirement of $37 \mathrm{~mW}$. The spread of values is expected as the thermal contact between the resistor and the wires will vary from piece to piece. In construction, a balance had to be reached between wrapping the wires tightly around the resistor, and not bending the twisting in a way that could open loops and increase the pick-up.

The heaters are only intended to be used for very brief periods so the heatload into the superfluid is not an issue. These tests were done in liquid helium, but tests of earlier prototypes in superfluid helium gave similar or lower required powers.

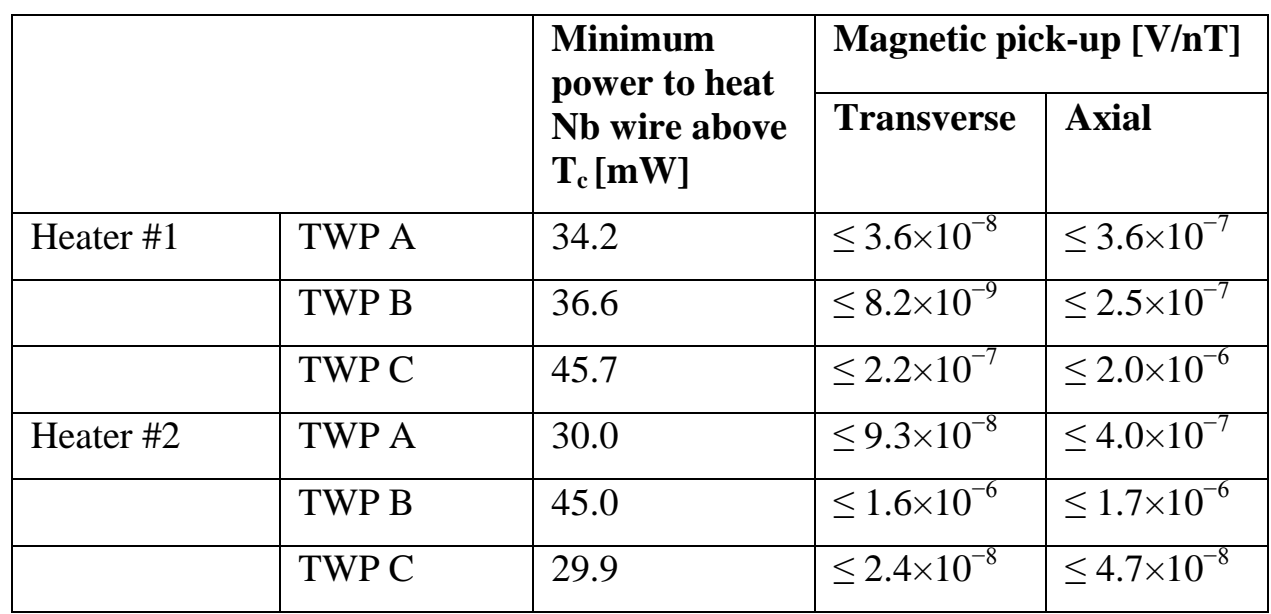

Table 4 The power required for the heater on two of the TWP assemblies to break the superconductivity of the NbTi wires, and the upper limits on the magnetic pick-up.

Given the results for the magnetic shielding factor of broken capillaries presented in Section 6.2 , it is reasonable to expect that the $\mathrm{Pb}$ cylinder housing the heater assembly is the likely weakest point in the magnetic shield. The shielding factor of the heater assembly could not be measured in the same way as that of the capillaries, as we could not remove the TWPs once installed. Instead we measured the magnetic pick-up of the heater assemblies by shorting the two ends on the TWP together, installing the heater at the centre of our Helmholtz coils, and 
testing this in the same way as the bare wire pairs as described in section 6. This pick-up factor depends on both the TWP twist geometry and the metal shield.

However our test setup was not designed to test these assemblies, and it was apparent from repeated measurements with the heater in different locations related to the Helmholtz coils that these tests were also sensitive to the pick-up of the bare ends of the TWPs at the other end of the assembly (despite being located away from the coils and shielding with additional $\mathrm{Pb}$ foil). Therefore these results, given for two assemblies in Table 4, are only an upper limit on the magnetic pick-up.

A pick-up of $10^{-6} \mathrm{~V} / \mathrm{nT}$ corresponds to an effective area of $6.7 \times 10^{-11} \mathrm{~m}^{2}$ (equivalent to $0.02 \%$ of the area of a single twist). Using this to calculate the error signal in the cryoEDM magnetometer due to the pick-up of a $1 \mu \mathrm{T}$ external fluctuation, using the procedure in section 3 , gives a value of $0.096 \mathrm{pT}$, just at the level of our target resolution.

\section{Conclusion}

The cryoEDM neutron electric dipole moment experiment requires magnetic shielding for twelve long twisted-wire-pairs which run between the SQUID sensors and magnetic pick-up loops. We have designed and built such a system using superconducting capillaries made from solder wire, where the flux core has been removed. Our system uses lengths of single and three-core solder wire, joined together, and incorporates a heater to allow the wires to be heated into their conducting state during operation in superfluid helium.

We have carried out a series of measurements of the magnetic pick-up of TWPs and the magnetic shielding factor of the solder capillaries; and the power required to heat the wires into a conducting state. The bare TWPs showed a response to applied transverse magnetic fields which is equivalent to an effective pick-up loop area of $5 \times 10^{-6} \mathrm{~m}^{2} / \mathrm{m}$ (equal to the area of 14 twists per metre). From this we determine that the superconducting capillaries in the cryoEDM experiment must provide a magnetic shielding factor of at least $2.0 \times 10^{4}$. Our measurements of these capillaries have shown the shielding factors are all greater than $10^{7}$. A break in a superconducting capillary typically reduces the shielding factor to $\sim 10$. When repaired with multiple layers of $\mathrm{Pb}$ foil this could be restored to $10^{3}-10^{4}$, but the level of success varied. An attempt to repair the capillary with a soldering iron restored the shielding factor to the previous value.

Measurements of the frequency spectra of the noise induced on shielded and unshielded TWPs have confirmed that the capillaries can reduce the low frequency fluctuations due to background magnetic noise to the level of the intrinsic SQUID noise.

Tests of the full assemblies for the cryoEDM experiment with three TWPs shielded by multiple sections of single and triple core capillaries have shown that the magnetic shielding properties are adequate and the internal heaters can heat the wires into a non-superconducting state with available power.

To summarise these tests have shown that capillaries made from solder wire are a high quality low-cost option for shielding twisted-wire pairs, and this design meets the requirements of the cryoEDM experiment. These tests also demonstrate the potential of using SQUIDs and superconducting wires to study the pick-up of twisted-wire pairs, which may be relevant to further applications.

\section{Acknowledgements}

We thank Mark Tucker (RAL) for drawing it to our attention to this method to remove flux from solder wires; Paul Pattinson for help setting up and running the apparatus to do this; Hans Kraus who designed the data acquisition hardware and software; and Michael McCann who also wrote much of the software. The cryoEDM experiment is a collaboration including 
the Universities of Oxford, Sussex, Swansea, Kure (Japan), the Rutherford Appleton Laboratory, and the Institut Laue-Langevin. This work was funded by STFC.

\section{References}

1. Improved Experimental Limit on the Electric Dipole Moment of the Neutron. Baker C A et al. Physical Review Letters 97 (2006) 131801.

DOI:10.1103/PhysRevLett.97.131801

2. A Proposal for a Cryogenic Experiment to Measure the Neutron Electric Dipole Moment (nEDM), Balashov S N et al, arXiv:0709.2428v1 [hep-ex]

3. CryoEDM: a cryogenic experiment to measure the neutron Electric Dipole Moment, C. A. Baker et al. International Conference on Neutron Scattering 2009. Journal of Physics Conference Series 251 012055. DOI:10.1088/1742-6596/251/1/012055

4. CryoEDM: A cryogenic experiment to measure the neutron electric dipole moment M. van der Grinten, Nucl. Instrum. Meth. A 611, 129 (2009). DOI:10.1088/17426596/251/1/012055

5. Measuring the electric dipole moment of the neutron: The cryoEDM experiment $\mathrm{C}$. Clarke et al., Europhysics Conference on High Energy Physics. PoS(EPS-HEP 2009) 376. http://pos.sissa.it/archive/conferences/084/376/EPS-HEP\%202009_376.pdf

6. Performance of an atomic mercury magnetometer in the neutron EDM experiment, K. Green et al. Nucl. Instrum. Meth. A 404 381-393 (1998). DOI: 10.1016/S01689002(97)01121-2

7. Apparatus for measurement of the electric dipole moment of the neutron using a cohabiting atomic-mercury magnetometer, C. A. Baker et al. Nucl. Instrum Meth. A 736 (2014) 184-203. DOI: 10.1016/j.nima.2013.10.005

8. A SQUID magnetometry system for a cryogenic neutron electric dipole moment experiment, S. Henry et al. To be submitted to Nucl. Instrum Meth. A.

9. SQUID magnetometry for the cryoEDM experiment - Test at LSBB, S Henry et al. JINST 3 (2008) P11003. DOI:10.1088/1748-0221/3/11/P11003

10. Magnetometry for cryoEDM. Michael McCann. D.Phil thesis, University of Oxford (2012).

11. Development of a SQUID-Based He-3 Co-Magnetometer Readout for a Neutron Electric Dipole Moment Experiment, Y. J. Kim and S. M. Clayton, IEEE Trans. Appl. Supercond. 23 (2013) 2500104, DOI: 10.1109/TASC.2012.2229773

12. Prediction of crosstalk involving twisted pairs of wires 1. Transmission-line model for twisted-wire pairs, C R Paul and J W McKnight, IEEE Trans. EMC 21 (1979) 92105. DOI: 10.1109/TEMC.1979.303751

13. The long-wavelength neutron spin-echo spectrometer IN15 at the Institut LaueLangevin, P. Schleger et al. Physica B 241 (1998) 164-165. DOI: 10.1016/S09214526(97)00539-5

14. Matter and Methods at Low Temperatures, F. Pobell. Springer-Verlag 1992.

15. http://www.inchem.org/documents/icsc/icsc/eics1063.htm

16. http://www.bhavanimetals.com/Solder_Wire_Rosin_Core_Solder.htm

17. Superconducting screening for SQUID flux transformers, C. M. Pegrum, Cryogenics 176 (1977) 369. DOI: 10.1016/0011-2275(77)90137-0

18. Inductance Calculation Techniques --- Part II: Approximations and Handbook Methods Power Control and Intelligent Motion, December 1999, Mark Thompson http://www.classictesla.com/download/indu_pt_2.pdf

19. Inductance Calculations: Working Formulas and Tables, Frederick W. Grover, Dover Publications, Inc., New York, (1946)

20. Superconducting magnetic shields for SQUID applications, J. R. Claycomb and J. H Miller Jr. Rev. Sci. Instrum. 70, 4562 (1999). DOI: 10.1063/1.1150113

21. Signal and noise theory for a dc SQUID amplifier, JM Martinis and J Clarke, J. Low Temp. Phys. 61 3-4 (1985) 227-236. DOI: 10.1007/BF00681633 